\title{
A Python Algorithm to Analyze Inelastic Neutron Scattering Spectra Based on the $y$-Scale Formalism
}

\author{
3 Claudia Scatigno, Giovanni Romanelli,* Enrico Preziosi, Matteo Zanetti, Stewart F. Parker, Svemir Rudić,
} ${ }_{4}$ Carla Andreani, and Roberto Senesi

Cite This: https://dx.doi.org/10.1021/acs.jctc.0c00790

Read Online

5 ABSTRACT: This paper presents a Python-based algorithm, 6 named INSCorNorm, to correct the inelastic neutron scattering 7 (INS) spectra for both sample and container self-shielding and to 8 normalize the experimental spectral intensity to an absolute 9 physical scale (barn/energy unit) facilitating the comparison with 10 computer simulations and interpretation. The algorithm is 11 benchmarked against INS measurements of $\mathrm{ZrH}_{2}$ performed on 12 the TOSCA spectrometer at the ISIS Facility. We also apply the 13 algorithm to the INS spectra from L-lysine, a system of broad 14 interest in biology and medicine, and we discuss how corrected INS data provide an experimental benchmark for theoretical 15 calculations of nuclear anisotropic displacement parameters in molecular systems. The total neutron sample cross section to use for 16 the self-shielding corrections is discussed, as well as the best approach to derive experimentally the cross section at the VESUVIO 17 spectrometer, together with the experimental value of the hydrogen nuclear mean kinetic energy, $\left\langle E_{\mathrm{k}}\right\rangle$. The algorithm is made 18 available to the neutron user community within the MANTID software.

\section{INTRODUCTION}

19 Neutron vibrational spectroscopy (inelastic neutron scattering, 20 INS) is a well-established experimental technique that probes 21 the atomic-scale dynamics of condensed matter systems. ${ }^{1,2}$ All 22 vibrational modes are allowed, and their spectral intensity 23 depends on the total scattering cross section and the amplitude 24 of motion of the atoms involved in the mode. The INS 25 physical process consists of an exchange of energy between 26 neutrons and nuclei resulting in the excitation of a phonon or a 27 lattice or molecular vibration in the sample under investigation. 28 Neutrons impinging on the system can lose a fixed amount of 29 energy in such a process, equal to the excitation energy of the 30 vibration, and the spectra resulting from an INS experiment are 31 representative of the vibrational density of states of the system.

32 INS spectrometers at spallation neutron sources, such as the 33 ISIS Pulsed Neutron and Muon Source (Didcot, UK), use 34 time-of-flight (TOF) methods for energy analysis. This 35 requires that either the energy of the incident or the scattered 36 neutron is known. These are known as direct and indirect 37 geometry spectrometers, respectively. Indirect geometry 38 spectrometers exploiting INS are generally used to observe 39 the low-energy transfer range, i.e., spectral features below 200 $40 \mathrm{meV}\left(1 \mathrm{meV} \simeq 8.065 \mathrm{~cm}^{-1}\right)$, where lattice and low-energy 41 vibrational modes manifest themselves. The TOSCA beamline 42 at ISIS has been a gold standard for INS for the last two 43 decades, as demonstrated by the TOSCA INS database ${ }^{3}$ and 44 the recent International Review. ${ }^{4}$ Moreover, the instrument capabilities have been significantly enhanced following the 45 installation of a neutron guide, ${ }^{5,6}$ the upgrade of the beryllium 46 filters, ${ }^{7}$ and the ability to exploit the empty pulse resulting from 47 ISIS TS2 operation, giving access to the elastic line and 48 extending the energy transfer range to as low as $-3 \mathrm{meV}$ (i.e., 49 the "anti-Stokes" side of the elastic line). ${ }^{7}$ Given the large cross 50 section of hydrogen ( 82.03 barn), the INS technique is 51 particularly suitable to study hydrogenous compounds where 52 the scattering of neutrons is dominated by hydrogen. $\mathrm{ZrH}_{2}$ is 53 often used as a reference material owing to its simple, 54 reproducible, and well-known behavior: it allows a full 55 assignment in terms of fundamental, overtone, ${ }^{8}$ and combina- 56 tion modes.

INS experimental results can be readily compared with the 58 INS spectra generated from single-molecule or periodic DFT 59 calculations. However, it is rare to express the experimental 60 data on an absolute intensity scale. The current practice is to 61 scale the calculated spectrum to match the experimental one. If 62 the experimental data were on an absolute scale, this would 63 remove an arbitrary procedure. The use of an absolute scale 64

Received: July 29, 2020 
65 would also facilitate data curation: instruments usually evolve 66 over the course of a typical 20 year lifespan and the 67 comparison of the spectra from different versions of the 68 same instrument is not necessarily straightforward. The 69 comparison of the spectra from nominally similar instruments 70 at different institutions is even more problematic. Recently, a 71 novel normalization procedure for INS data has been 72 suggested, ${ }^{10}$ which is based on the $y$-scaling ${ }^{11,12}$ formalism 73 within the framework of the impulse approximation (IA). ${ }^{13,14}$ 74 The latter is valid at high values of energy transfer, $E_{\mathrm{T}}$, and 75 wave vector, $Q .{ }^{12,14}$ This is the deep inelastic neutron 76 scattering (DINS) regime, ${ }^{15,16}$ where the VESUVIO instru77 ment $^{17-22}$ at ISIS operates to measure nuclear mean kinetic 78 energies, $\left\langle E_{\mathrm{k}}\right\rangle$, and momentum distributions, $n(p)$, of light ${ }^{14}$ 79 and heavy nuclei. ${ }^{15}$ This novel procedure requires knowledge 80 of the energy-dependent total neutron cross section of the 81 sample under investigation and the value of $\left\langle E_{\mathrm{k}}\right\rangle$.

82 The energy-dependent total neutron cross sections, $\sigma(E)$, is 83 a physical quantity ${ }^{23-26}$ related to the structure and dynamics 84 of a sample at the microscopic scale. Measurements of $\sigma(E)$ 85 can be performed using broad-band neutron transmission at 86 the VESUVIO spectrometer ${ }^{27-29}$ in the energy range meV$87 \mathrm{keV}$, concurrently with the DINS experiment.

88 In this paper, we present the algorithm INSCorNorm that 89 allows container and self-shielding corrections of INS data 90 collected at the TOSCA spectrometer. It provides a physical 91 and intrinsic normalization of the data based on the discussion 92 in ref 10. The latter introduces the use of an approximated 93 model for $\sigma(E)$, namely, the free hydrogen gas (FHG), and an 94 approximate estimate for the hydrogen $\left\langle E_{\mathrm{k}}\right\rangle$ for the normal95 ization. Here, we go beyond these approximations by 96 proposing the use of both the experimental quantities, $\sigma(E)$ 97 and $\left\langle E_{\mathrm{k}}\right\rangle$, measured on the VESUVIO spectrometer. An 98 additional model for $\sigma(E)$ for hydrogen in organic molecules ${ }^{25}$ 99 is also implemented within the algorithm, to be employed for 100 similar samples in cases where the experimental cross section is 101 not available. We benchmark the new algorithm to the case of $102 \mathrm{ZrH}_{2}$, a suitable analyte wherein the hydrogen dynamics can be 103 approximated using an isotropic and harmonic potential with a 104 fundamental transition energy of ca. $140 \mathrm{meV} .^{30,31}$ Moreover, 105 we apply the algorithm to the case of the essential amino acid 106 L-lysine, ${ }^{32}$ a compound of interest in both biology ${ }^{33}$ and soft 107 matter. $^{34}$

\section{MATERIALS AND METHODS}

108 Experiments were performed on commercially available 109 powder samples of $\mathrm{ZrH}_{2}$. Two samples with different amounts 110 and thicknesses were used, as shown in Table 1. Additional 111 measurements on L-lysine, discussed in Section 4.1, shared the 112 same experimental details presented below.

Table 1. Experimental Details for the $\mathrm{ZrH}_{2}$ and $\mathrm{L}$-Lysine Samples: Thickness of the Sample, Mass of the Samples, and Integrated Proton Charge, IPC $(\mu \mathrm{Ah})$, Related to the TOSCA and VESUVIO Measurements

\begin{tabular}{|lccc|}
\hline \multicolumn{1}{|c}{ parameter } & sample 1 & sample 2 & L-lysine \\
thickness $(\mathrm{cm})$ & 0.100 & 0.244 & 0.100 \\
mass $(\mathrm{g})$ & 5.357 & 9.434 & 2.698 \\
$\mathrm{IPC}_{\mathrm{TOSCA}}(\mu \mathrm{Ah})$ & 169 & 600 & 200 \\
$\mathrm{IPC}_{\mathrm{VESUVIO}}(\mu \mathrm{Ah})$ & 3600 & 3600 & 3060 \\
\hline
\end{tabular}

The measurements were carried out using TOSCA for INS ${ }^{31} 113$ (Figure 1) and $\mathrm{VESUVIO}^{35}$ for neutron transmission and $114 \mathrm{f1}$

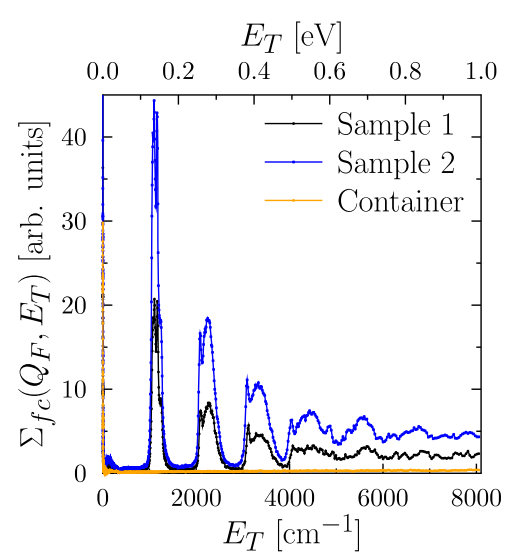

Figure 1. Experimental filled-container INS spectra, $\Sigma_{\mathrm{fc}}\left(Q_{\mathrm{F}}, E_{\mathrm{T}}\right)$ of both $\mathrm{ZrH}_{2}$ samples (sample 1, black line, sample 2, blue line). The spectrum from the empty container, $\Sigma_{\mathrm{ec}}\left(Q_{\mathrm{F}}, E_{\mathrm{T}}\right)$, is also shown (orange line).

DINS. Standard flat Al cells, $4.0 \times 4.8 \mathrm{~cm}^{2}$ in size, were used 115 for both experiments. Taking into account the TOSCA beam 116 size, ${ }^{5}$ the amounts of sample in the beam were 98 and $78 \%$ for 117 sample 2 and sample 1 , respectively, as determined by neutron 118 radiography.

The TOSCA instrument is an inverted-geometry TOF 120 spectrometer with a wide range of energy transfer $\left(E_{\mathrm{T}}\right), 121$ between -3 and $1000 \mathrm{meV}$, and angular coverage over two 122 fixed dynamic trajectories (i.e., back scattering and forward 123 scattering). Neutrons scattered from the sample are Bragg- 124 reflected from a pyrolytic graphite analyser (HOPG) toward 125 the detectors, with a final average neutron energy of $E_{\mathrm{f}} \simeq 4126$ $\mathrm{meV}$. Experiments on TOSCA are routinely performed by 127 placing the sample into a top-loading closed-cycle refrigerator 128 (CCR) kept at a base temperature of about $10 \mathrm{~K}$ and in the 129 environment of $10 \mathrm{mbar}$ of helium gas used to facilitate the 130 sample cooling via heat exchange with the CCR walls. The INS 131 detector banks have five analyser modules in back-scattering 132 direction $\left(\theta_{\mathrm{B}} \simeq 135^{\circ}\right)$ and five in the forward-scattering $\left(\theta_{\mathrm{F}} \simeq 133\right.$ $\left.45^{\circ}\right)$ direction. The instrument is characterized by its $17 \mathrm{~m} 134$ primary flight path and approximately $4 \times 4 \mathrm{~cm}^{236}$ beam size at 135 the sample position, ${ }^{7}$ taken as the full width at half-maximum 136 of the beam profile.

The VESUVIO spectrometer is a TOF instrument that 138 records neutrons scattered between 30 and $70^{\circ}$ in forward 139 scattering and between 130 and $170^{\circ}$ in back scattering. ${ }^{27,37-39} 140$ Neutrons scattered along the hydrogen recoil line cover the $Q_{141}$ range 30-200 $\AA^{-1}$. DINS experiments on VESUVIO allow the 142 measurement of atomic momentum distributions and nuclear 143 mean kinetic energies in condensed matter systems ${ }^{15,16}$ (see 144 Section 3.2). For concurrent neutron transmission measure- 145 ments, ${ }^{28}$ the monitors, Li-doped glass scintillators, are located 146 at $13.43 \mathrm{~m}$ (after the sample) and $8.60 \mathrm{~m}$ (before the sample) 147 from the moderator, along the beam direction. The sample is 148 placed in a CCR at $11 \mathrm{~m}$ from the moderator. Transmission 149 and DINS experiments on VESUVIO were performed using 150 the same samples (and containers) following the measure- 151 ments on TOSCA, and the transmission spectra were analyzed 152 in the same range of incident energies as for the INS spectra. 153 


\section{CORRECTION AND NORMALIZATION ALGORITHM}

154 The INSCorNorm algorithm corrects the INS spectra for 155 empty container and sample self-shielding and finally normal156 izes the spectra to a physical scale of barn/energy unit. The 157 latter quantity corresponds to the double differential scattering 158 cross section averaged over the angular solid angle related to 159 the position of the detectors. In the case of TOSCA-type 160 instruments, this corresponds, in practice, to two narrow 161 scattering angles, one for the forward and one for the backward 162 detector banks. However, the algorithm can be easily 163 generalized so as to normalize each detector separately, thus 164 providing a punctual angular information for instruments with 165 different detector geometries.

166 In this section, a detailed description of the corrections is 167 provided, then the normalization procedure is discussed, and 168 finally an explanation on how the theory is translated into the 169 proposed algorithm is given. The procedure was suggested in 170 ref 10 from where we adopt a simplified notation. The signal 171 from the sample within the container can be expressed as

$$
\begin{aligned}
\Sigma_{\mathrm{fc}}\left(E_{\mathrm{T}}\right)= & T_{\mathrm{c}}(0) S\left(\theta_{\mathrm{F}, \mathrm{B}}, E_{\mathrm{i}}, E_{\mathrm{f}}\right) T_{\mathrm{c}}\left(\theta_{\mathrm{F}, \mathrm{B}}\right) \Sigma_{\mathrm{s}}\left(Q_{\mathrm{F}, \mathrm{B}}, E_{\mathrm{T}}\right) \\
& +\left(T_{\mathrm{s}}\left(0, E_{\mathrm{i}}\right) T_{\mathrm{c}}(0)+T_{\mathrm{s}}\left(\theta_{\mathrm{F}, \mathrm{B}}, E_{\mathrm{f}}\right) T_{\mathrm{c}}\left(\theta_{\mathrm{F}, \mathrm{B}}\right)\right) W \\
& \left(Q_{\mathrm{F}, \mathrm{B}}, E_{\mathrm{T}}\right)
\end{aligned}
$$

173 while, for the empty container, the experimental signal can be 174 expressed as

$$
175 \quad \Sigma_{\mathrm{ec}}\left(E_{\mathrm{T}}\right)=\left(T_{\mathrm{c}}(0)+T_{\mathrm{c}}\left(\theta_{\mathrm{F}, \mathrm{B}}\right)\right) W\left(Q_{\mathrm{F}, \mathrm{B}}, E_{\mathrm{T}}\right)
$$

176 Here, $\Sigma_{s}\left(Q_{F, B}, E_{T}\right)$ is the macroscopic self-scattering law for 177 the sample, including the convolution with instrumental 178 resolution, $S\left(Q_{F, B}, E_{T}\right)$ is the sample self-shielding function, 179 while $T_{s}(\theta, E)$ and $T_{c}(\theta)$ are the sample and container 180 attenuation functions, respectively. Finally, $W\left(Q_{\mathrm{F}, \mathrm{B}}, E_{\mathrm{T}}\right)$ is the 181 signal coming from one container wall (not corrected for its 182 own self-shielding). In this procedure, the sample transmission 183 is taken as a function of the neutron energy, either before or 184 after the scattering event, while the transmission of the empty 185 container is taken as a constant function of the neutron energy. 186 One should notice that, for the majority of neutron 187 experiments, the container is manufactured either with $\mathrm{Al}$ or 188 with other low-scattering materials, and its attenuation for $\mathrm{eV}$ 189 neutrons is of the order of few percent. For cold and thermal 190 neutrons, the features in the attenuation function are primarily 191 driven by Bragg edges that are considered negligible for such 192 thin containers. Finally, differing from ref 10, any multiple 193 scattering contributions at this stage have been neglected. 194 Figure 1 shows the experimental signals for the two $\mathrm{ZrH}_{2}$ 195 samples in the container, $\Sigma_{\mathrm{fc}}$ and for the empty container, $\Sigma_{\mathrm{ec}}$. 196 3.1. Corrections for Attenuation and Self-Shielding. 197 The energy-dependent attenuation of neutrons through the 198 sample is a function of the energy-dependent cross section and 199 of the sample geometry. It can be obtained in neutron 200 transmission measurements interpreted via the Beer-Lambert 201 law, whereby the transmission spectrum as a function of the 202 incident neutron energy, $T(E)$, is related to $\sigma(E)$ as

203

$$
T(E)=\exp \left(-n_{\mathrm{H}} \sigma(E)\right)
$$

204 with the areal density of hydrogen atoms $\left(n_{\mathrm{H}}\right)$ defined from the 205 mass of sample inserted in the container $(m)$, the container 206 surface $(A)$, the molar mass of the same formula unit to which the cross section refers to $(M)$, and the number of hydrogen 207 atoms per formula unit $\left(N_{\mathrm{H}}\right)$,

$$
n_{\mathrm{H}}=\frac{N_{\mathrm{H}} m}{M A \mu}
$$

If $m$ is expressed in $\mathrm{g}, A$ in $\mathrm{cm}^{2}$, and $M$ in amu, one can use $\mu=210$ 1.66054 to obtain $n_{\mathrm{H}}$ in $\mathrm{barn}^{-1}$. Figure 2 shows the $211 \mathrm{f} 2$

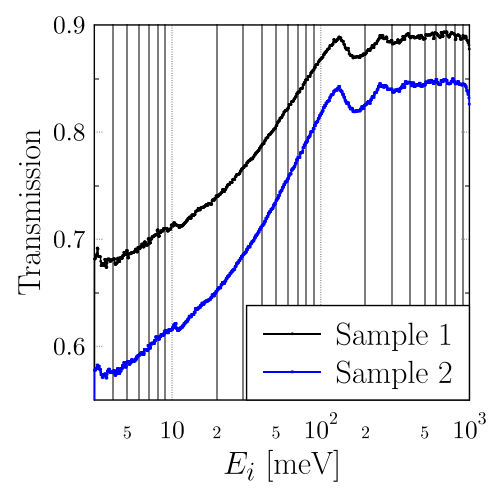

Figure 2. Experimental neutron transmission of $\mathrm{ZrH}_{2}$ as a function of the incident neutron energy, as measured on the VESUVIO spectrometer: sample 1 (black line) and sample 2 (blue line).

experimental neutron transmission from the two samples of 212 $\mathrm{ZrH}_{2}$, as measured on VESUVIO. By comparison with the INS 213 spectra shown in Figure 1, one can appreciate how the features 214 in $T(E)$, thus in $\sigma(E)$, are closely related to the probability to 215 excite a vibration in the INS spectra. The low-energy region is 216 dominated by the incoherent elastic scattering from hydrogen; 217 the following features at ca. 140, $280 \mathrm{meV}$, etc., correspond, 218 within the framework of the multiphonon expansion of the 219 dynamic structure factor, to the 1-phonon and 2-phonons 220 terms, respectively.

221

Qualitative inspection of Figure 2 delivers two clear 222 messages. First, the transmission function, thus the sample 223 attenuation, is sample-dependent: the position and intensity of 224 the features in the total scattering cross section are strictly 225 related to the vibrations taking place at the microscopic level. 226 These are especially noticeable for hydrogenous samples. 227 Second, by comparing the spectral intensity for the two 228 samples with the value of $\mathrm{ZrH}_{2}$ cross section available in the 229 literature, one can notice how the Beer-Lambert law only 230 applies if the bulk density of the samples, $5.6 \mathrm{~g} / \mathrm{mL}$, is replaced 231 with a preparation-dependent powder density. As a con- 232 sequence, in order to perform as accurate corrections as 233 possible of the INS data, an experimental determination of the 234 transmission is needed.

235

One can also notice that eqs 1 and 2 require the 236 determination of both the transmission of the sample for 237 incident neutrons impinging on the sample at $\theta=0$ and for 238 scattered neutrons at a generic angle $\theta$. While the neutron 239 transmission measurement allows the direct determination of 240 $T(0, E) \equiv T(E)$, one can obtain $T(\theta, E)$ assuming that the 241 values of density and cross section remain the same for 242 scattered neutrons, yet the thickness of the neutron path in the 243 sample increased. Therefore, the following relation is used in 244 the algorithm:

$$
T(\theta, E)=\exp \left(\frac{\ln T(0, E)}{\cos \theta}\right)
$$


247 Considering that in many cases it is not possible to 248 determine the experimental cross section or, more importantly, 249 the experimental transmission of the sample in the INS 250 experiment, three options are available:

251

252

253

254

255

256

284 One should notice that approximated values of $\left\langle E_{\mathrm{k}}\right\rangle$ and $T^{*}$ 285 can be obtained self-consistently from a simulated vibrational 286 density of states, $g\left(E_{\mathrm{T}}\right)$ as ${ }^{10,14,15}$

287

$$
\frac{3}{2} k_{\mathrm{B}} T^{*}=\left\langle E_{\mathrm{k}}\right\rangle=\frac{1}{4} \int E_{\mathrm{T}} g\left(E_{\mathrm{T}}\right) \operatorname{coth}\left(\frac{E_{\mathrm{T}}}{2 k_{\mathrm{B}} T}\right)
$$

288 As far as the self-shielding correction is concerned, the 289 following analytical approximation for a flat-geometry sample ${ }^{45}$ 290 have been used, respectively, for forward scattering (trans291 mission geometry):

$$
292 \quad S\left(\theta_{\mathrm{F}}, E_{\mathrm{i}}, E_{\mathrm{f}}\right)=\frac{T\left(0, E_{\mathrm{i}}\right)-T\left(\theta_{\mathrm{F}}, E_{\mathrm{f}}\right)}{\log \left(T\left(0, E_{\mathrm{i}}\right)\right)-\log \left(T\left(\theta_{\mathrm{F}}\right), E_{\mathrm{f}}\right)}
$$

293 and back scattering (reflection geometry):

$$
S\left(\theta_{\mathrm{B}}, E_{\mathrm{i}}, E_{\mathrm{f}}\right)=\frac{T\left(0, E_{\mathrm{i}}\right) T\left(\theta_{\mathrm{B}}, E_{\mathrm{f}}\right)-1}{\log \left(T\left(0, E_{\mathrm{i}}\right)\right)+\log \left(T\left(\theta_{\mathrm{B}}\right), E_{\mathrm{f}}\right)}
$$

295 If the transmission function is obtained from a model cross 296 section, one needs to provide an estimate of the areal density. 297 If the experimental value of the transmission function is 298 available, the areal density is included in the transmission data.
While the previous formalism holds in the case of flat-geometry 299 containers, generally used on indirect-geometry INS spec- 300 trometers, the MANTID environment includes other Monte- 301 Carlo-based algorithms to evaluate the self-attenuation 302 correction for other sample geometries that can be used 303 within INSCorNorm if necessary.

304

3.2. Normalization of the Spectra. The normalization 305 procedure follows and expands on what was introduced in ref 306 10. It assumes the validity of both the multiphonon 307 expansion ${ }^{46}$ for thermal neutrons and the IA for epithermal 308 neutrons, in the range of energy transfer $600 \mathrm{meV}-1 \mathrm{eV}$. $\quad 309$

Within the IA, ${ }^{1,15}$ the nucleus is considered a non- 310 interacting particle recoiling freely after a scattering event. 311 The energy transfer, $E_{\mathrm{T}}$, is therefore centered around the recoil 312 energy, $E_{R}$, inversely proportional to the nuclear mass, $M$, and 313 proportional to the square of the wave vector, $Q$

$$
E_{\mathrm{T}}=\frac{\hbar^{2} Q^{2}}{2 M}+\frac{\hbar \vec{Q} \cdot \vec{p}}{2 M}=E_{\mathrm{R}}+\frac{\hbar^{2} Q}{M} y
$$

where $\vec{p}$ is the momentum of the nucleus before the scattering 316 event, and $y=\vec{p} \cdot \hat{Q} / \hbar$ is its projection along the direction of 317 the momentum transfer. Before the scattering event, the 318 dynamics of the nucleus depends on the molecular or 319 crystalline vibrational modes, with a mean kinetic energy 320 $\left\langle E_{\mathrm{k}}\right\rangle=3 \hbar^{2} \sigma_{\mathrm{p}}^{2} / 2 M$, with $\sigma_{\mathrm{p}}$ being the standard deviation of the 321 nuclear momentum distribution $\sigma_{\mathrm{p}}^{2}=\left\langle y^{2}\right\rangle$. The nuclear 322 momentum distribution can be accessed in a DINS experiment 323 via the neutron Compton profile (NCP), ${ }^{14,15}$ and for a system 324 described by a harmonic and isotropic potential, it can be 325 written as

$$
S_{\mathrm{IA}}\left(Q, E_{\mathrm{T}}\right)=\frac{M}{\hbar^{2} Q} J(y)=\frac{M}{\hbar^{2} Q} \frac{\exp \left(-\frac{y^{2}}{2 \sigma_{\mathrm{p}}^{2}}\right)}{\sqrt{2 \pi \sigma_{\mathrm{p}}^{2}}}
$$

By replacing $y=\frac{M}{\hbar \mathrm{Q}}\left(E_{\mathrm{T}}-E_{\mathrm{R}}\right)$, one gets the familiar ${ }_{328}$ expression of the dynamic structure factor for a gas of free 329 nuclei

$$
S_{\mathrm{IA}}\left(Q, E_{\mathrm{T}}\right)=\frac{\exp \left(-\frac{3\left(E_{\mathrm{T}}-E_{\mathrm{R}}\right)^{2}}{8\left(E_{\mathrm{k}} E_{\mathrm{R}}\right.}\right)}{\sqrt{8 \pi E_{\mathrm{R}}\left\langle E_{\mathrm{k}}\right\rangle / 3}}=\frac{\exp \left(-\frac{\left(E_{\mathrm{T}}-E_{\mathrm{R}}\right)^{2}}{2 E_{\mathrm{R}} \hbar \omega_{0}}\right)}{\sqrt{2 \pi E_{\mathrm{R}} \hbar \omega_{0}}}
$$

where the last equality only holds for a single harmonic 332 oscillator of frequency $\omega_{0}$, i.e., for a harmonic and isotropic 333 potential. This is a satisfactory approximation for $\mathrm{ZrH}_{2}$, where 334 $\hbar \omega_{0} \simeq 140 \mathrm{meV}$.

The connection between the IA and the multiphonon 336 expansion was already discussed by Watson ${ }^{47}$ for a similar 337 system with only one phonon frequency, with the result 338

$$
S_{\mathrm{MP}}\left(Q, E_{\mathrm{T}}\right)=e^{-E_{\mathrm{R}} / \hbar \omega_{0}} \sum_{n} \frac{1}{n !}\left(\frac{E_{\mathrm{R}}}{\hbar \omega_{0}}\right)^{n} \delta\left(E_{\mathrm{T}}-n \hbar \omega\right)
$$

A comparison of the two models is provided in Figure 3 for a $340 \mathrm{fz}$ series of values of the final energy $E_{\mathrm{f}}$ on an inverted-geometry 341 spectrometer, with the two special cases of TOSCA (black 342 lines), $E_{\mathrm{f}} \simeq 3 \mathrm{meV}$ and VESUVIO (gold lines), with $E_{\mathrm{f}} \simeq 5 \mathrm{eV}$. 343 On the top panel of the figure, as the final energy increases, 344 both $E_{\mathrm{T}}$ and $Q$ increase, and $S_{\mathrm{IA}}$ shows a peak, proportional to 345 $J(y)$, centred about $E_{\mathrm{R}}$ and broadened by $\left\langle E_{\mathrm{k}}\right\rangle$. The black line 346 


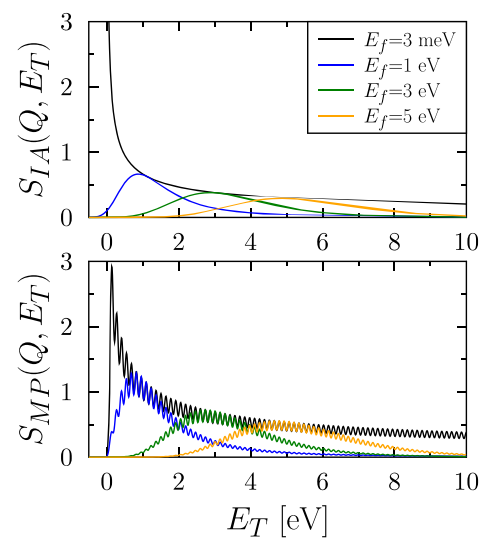

Figure 3. Dynamic structure factor in the impulse approximation (top) and in the multiphonon expansion (bottom) as a function of the energy transfer $E_{\mathrm{T}}=E_{\mathrm{i}}-E_{\mathrm{f}}$ for a series of values of the final energy $E_{\mathrm{f}}$.

$347\left(E_{\mathrm{f}}=3 \mathrm{meV}\right)$ is not representative of experimental data, and it 348 is only an extrapolation of eq 11 . In fact, the spectra in this case 349 are better represented by eq 13, whose graphical representation 350 is reported on the bottom panel of Figure 3. By comparison of 351 the results from the two equations, one can notice how the 352 modulation of the overtones in the multiphonon expansion of $353 S_{\mathrm{MP}}$ follows the same trend as $S_{\mathrm{IA}}$, for all the values of $E_{\mathrm{f}}$. While 354 the single overtones are still visible in the analytic calculation 355 of Figure 3 (bottom), in the experimental spectra, owing to the 356 instrument resolution, the overtones soon combine to form a 357 continuous and flat signal on an INS spectrometer, as one can 358 observe on the right side of Figure 1, or a Gaussian-like peak 359 on a DINS spectrometer.

360 The ideal equations valid in the IA are generally corrected 361 for final state effects ${ }^{48}$ that take into consideration the fact that 362 neither $E_{\mathrm{T}}$ nor $Q$ is infinite in practice. Here, the additive 363 corrections to the $\mathrm{NCP}^{10,15}$ method are adopted:

364

$$
\begin{aligned}
S_{\mathrm{IA}, \mathrm{FSE}}\left(Q, E_{\mathrm{T}}\right)= & S_{\mathrm{IA}}\left[1+\frac{b_{3}}{Q} H_{3}(x)+\frac{b_{4}}{Q^{2}} H_{4}(x)\right. \\
& \left.+\frac{b_{6}}{Q^{2}} H_{6}(x)\right]
\end{aligned}
$$

365 where the parameters $b_{n}$ are functions of $\sigma_{\mathrm{p}}$ in the harmonic 366 approximation, ${ }^{10}$ and $H_{n}(x)$ are the Hermite polynomials, with ${ }_{367} x=y / \sqrt{2} \sigma_{\mathrm{p}}$

368 Finally, one can express the self-scattering law as

369

$$
\Sigma_{\mathrm{IA}, \mathrm{FSE}}\left(Q, E_{\mathrm{T}}\right)=\frac{\sigma_{\mathrm{b}}}{4 \pi} \sqrt{\frac{E_{\mathrm{f}}}{E_{\mathrm{i}}}} S_{\mathrm{IA}, \mathrm{FSE}}\left(Q, E_{\mathrm{T}}\right)
$$

370 Following the discussion in ref 10 , one can normalize the INS 371 spectra by imposing the requirement that the experimental 372 intensity in the high energy-transfer region has the same 373 intensity as in eq 11

$$
374 \int_{E_{\min }}^{E_{\max }} \Sigma_{\mathrm{s}}\left(Q, E_{\mathrm{T}}\right) d E_{\mathrm{T}}=\int_{E_{\min }}^{E_{\max }} \Sigma_{\mathrm{IA}, \mathrm{FSE}}\left(Q, E_{\mathrm{T}}\right) d E_{\mathrm{T}}
$$

375 Considering that this equation only depends on the value of $376 \sigma_{\mathrm{p}}$ or equivalently of $\left\langle E_{\mathrm{k}}\right\rangle$, one can convert the intensity of the 377 INS spectra to a physical scale using the results of a DINS 378 experiment.
3.3. Algorithm Implementation. The aforementioned 379 procedures have been put together in a unique algorithm 380 specifically developed in Python to allow its implementation in 381 MANTID. ${ }^{49}$ This choice has been made to allow the neutron 382 scattering community to take advantage of it, since the script 383 presents an user-friendly graphical user interface.

384

The algorithm, as depicted in Figure 4 via a use case $385 \mathrm{f4}$ diagram, can be divided into four blocks:

386

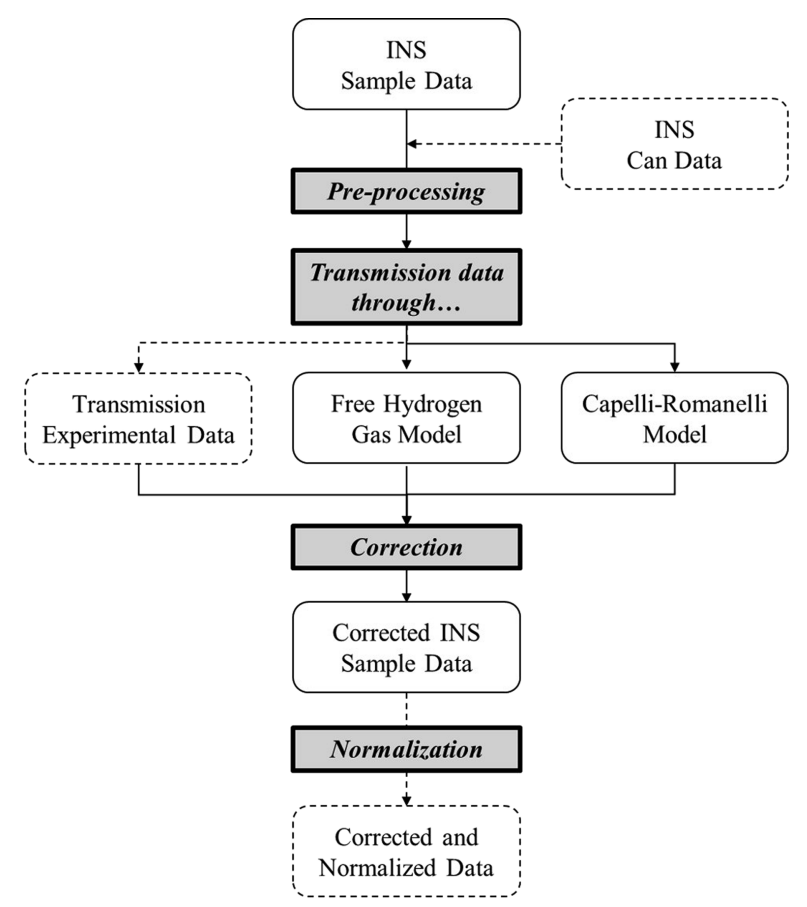

Figure 4. Use case diagram of the algorithm, depicting the workflow, the data process, and the four main steps, which are highlighted with a gray background. Optional procedures are shown with dashed lines.

1. INS data import and preprocessing;

2. Transmission data import or transmission modeling; 388

3. Self-shielding correction; 389

4. Normalization using $y$-scale formalism.

In INS data import and preprocessing, the INS experimental 391 data are loaded into the script. Depending on the experimental 392 conditions, the user can also load the data (if available) of the 393 INS measurement of the empty sample. If these are provided, 394 in the correction phase, the container signal is subtracted from 395 the experimental data related to the sample enclosed in the 396 container, in order to remove additional contributions 397 provided by the container itself. The container, even if 398 typically made of aluminium that guarantees nearly complete 399 transmission (about 99\%), adds an additional attenuation 400 effect on the measurement.

Before being processed, both the sample and the (optional) 402 container data undergo unit conversion because the analyses in 403 the algorithm are provided with energy transfer in meV. The 404 data are then converted to incident energy, $E_{\mathrm{i}}=E_{\mathrm{T}}+E_{\mathrm{f}} . \quad 405$

In transmission data import or transmission modeling, for 406 the correction procedure, transmission data must be fed into 407 the script. This can be carried out in two different ways (see 408 Figure 4): (a) if transmission data are available, they can be 409 used as a data input to the script and (b) if transmission data 410 are not available, the script allows the user to select either the 411 
412 FHG model or the Capelli-Romanelli model. The user needs 413 to provide the sample-specific areal density using eq 4 . Using 414 any of these two models, the algorithm produces a workspace 415 with the tabulated transmission spectrum.

416 In self-shielding correction, once the transmission spectrum 417 is available, the algorithm corrects the INS data of the sample 418 according to eqs 1 and 2 . The measured signals are then 419 corrected for sample self-shielding and for both sample and 420 container attenuation.

421 In normalization using $y$-scale formalism, finally, the spectra 422 can be normalized using the $y$-scaling formalism. The user can 423 provide either an experimental value obtained in a DINS 424 experiment or a theoretical value predicted, for example, in a 425 DFT-based simulation and using eq 7. The script requires 426 some additional parameters such as the nuclear mean kinetic 427 energy of hydrogen, the number of hydrogen atoms per unit 428 cell of the sample, and the energy range for the normalization 429 procedure.

430 After these corrections, INS data coming from different 431 experimental conditions both from the sample and the 432 instrumentation standpoints can be compared for further 433 analysis.

\section{RESULTS AND DISCUSSION}

434 Results of the DINS data from $\mathrm{ZrH}_{2}$ provide the parameters 435 needed for the normalization of the INS spectra. Figure 5

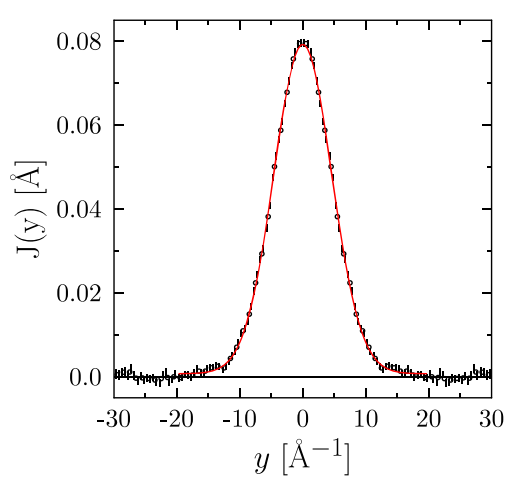

Figure 5. Neutron Compton profile of hydrogen in $\mathrm{ZrH}_{2}$ (sample 1, black error bars) at $300 \mathrm{~K}$ as measured on VESUVIO, together with its best fit (red solid line).

436 shows the NCP of hydrogen in sample 1 after the standard 437 data reduction of the DINS data using MANTID, ${ }^{50}$ after 438 corrections for environmental- ${ }^{51,52}$ and sample-dependent 439 backgrounds, ${ }^{19,53,54}$ and convolution with the instrument 440 resolution. ${ }^{55-57}$ The analysis provides a value of the total 441 mean kinetic energy of $\left\langle E_{\mathrm{k}}\right\rangle=113 \pm 2 \mathrm{meV}$, corresponding to 442 a standard deviation $\sigma_{\mathrm{p}}=4.22 \pm 0.02 \AA^{-1}$. The value is in 443 excellent agreement with values previously reported in the 444 literature. $^{22,58}$ Concurrent measurements of neutron trans445 mission on VESUVIO (see also Figure 2) are reported for the $4461 \mathrm{~mm}$-thick sample in Figure 6 to be compared with the two 447 models. One should first notice how, for neutron energies 448 approaching the epithermal limit $\left(E_{\mathrm{i}} \rightarrow 1 \mathrm{eV}\right)$, the model 449 transmission functions overlap to the experimental one. 450 Theoretically, at epithermal energies, the cross section is no 451 longer affected by phonons or internal vibrations. On a 452 practical side, the model transmissions overlap to the 453 experimental one only if the real sample density is known. 454 As a first guess, the experimenter can define the density from

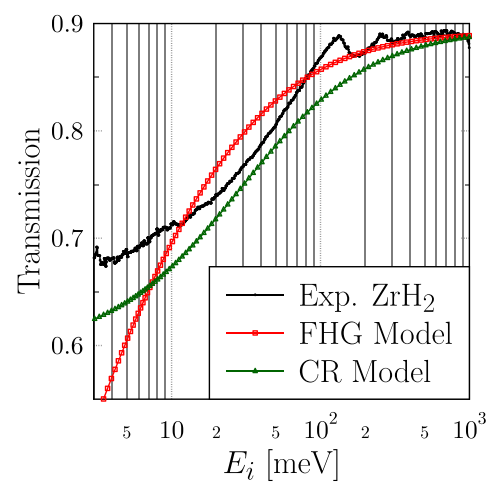

Figure 6. Comparison of the three cross section models of the experimental TOSCA and VESUVIO data of $\mathrm{ZrH}_{2}$ of $1 \mathrm{~mm}$ in thickness.

the loaded mass of sample in a given volume of the container. 455 However, especially for powder samples, one can expect the 456 sample to rearrange within the container, possibly moving 457 toward the bottom. This was the case in the present 458 experiment where, as opposed to usual experiments, we 459 could monitor the sample distribution within the container 460 using neutron radiography (not shown here). From the 461 radiographs, in the case of the $1 \mathrm{~mm}$-thick sample, we could 462 conclude that the container height was filled only to about 463 $78 \%$, and we corrected the sample density accordingly. $\quad 464$

For cold and thermal neutrons, Figure 6 shows drastic 465 differences in the models compared to the experimental 466 spectrum. The model by Capelli and Romanelli (CR) provides 467 a cross section that is always larger than that of $\mathrm{ZrH}_{2}$. This is 468 because organic molecules have more vibrational modes than 469 the 1-phonon density of states of $\mathrm{ZrH}_{2}$. Therefore, the 470 contributions from the multiphonon expansion overlap, and 471 individual contributions are no longer recognized. Moreover, 472 in the limit $E_{\mathrm{i}} \rightarrow 0$, both the CR and experimental functions 473 tend to constant values, driven by the bound scattering cross 474 section of hydrogen. On the other hand, the FHG model 475 suffers from a divergent cross section for vanishing neutron 476 energies, as expected of free atoms not bound to a lattice or 477 molecule.

478

Figure 7 shows the experimental spectra from sample 1 and $479 \mathrm{f} 7$ sample 2 after the corrections and normalization. The two 480 spectra satisfactorily overlap, showing that the self-shielding 481 corrections were successful. One should notice that the 482

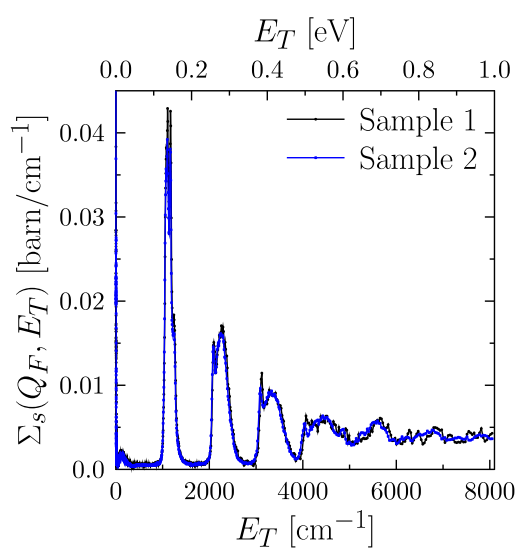

Figure 7. Corrected and normalized INS spectra of $\mathrm{ZrH}_{2}$ from sample 1 (black line) and sample 2 (blue line). 
483 intensity of the peak of the first phonon for sample 1 would 484 have been underestimated by ca. $10 \%$ if no such corrections 485 would have been performed. A closer inspection shows that, 486 for the thinner sample (sample 1), the peak features are 487 sharper than for the thicker sample (sample 2). This can be 488 related to the presence of multiple scattering in the sample 489 with the higher scattering power. The double scattering can be 490 approximated, as a first approximation, to the convolution of 491 the single-scattering spectrum and the elastic line. Therefore, 492 the result is a replica of the single-scattering spectrum, well 493 represented by sample 1 , yet with less-defined features. In this 494 scenario, the presence of multiple scattering does not invalidate 495 the normalization procedure and justifies a posteriori the 496 decision to neglect multiple-scattering contributions in eq 1 .

497 4.1. Application to L-Lysine. Lysine is an essential amino 498 acid that mammals must acquire from food. The interactions of 499 L-lysine in biological systems are largely dependent on the 500 formation of hydrogen bonds involving the aliphatic side chain 501 and especially the $\mathrm{NH}_{2}$ group. ${ }^{59,60}$ Such intermolecular 502 interactions are particularly evident at low values of $E_{\mathrm{T}}$, thus 503 making a proper correction procedure of INS data a 504 fundamental step when comparing to computer simulations.

505 As an additional test of the correction and normalization 506 procedure, we performed measurements on one L-lysine 507 sample loaded in the same type of TOSCA container with a 508 nominal hydrogen areal density $n_{\mathrm{H}}=0.0081 \mathrm{barn}^{-1}$. The INS 509 spectrum from this sample, corrected and normalized using the 510 INSCorNorm algorithm, is reported in the bottom panel of 511 Figure 8. In particular, this spectrum corresponds to the

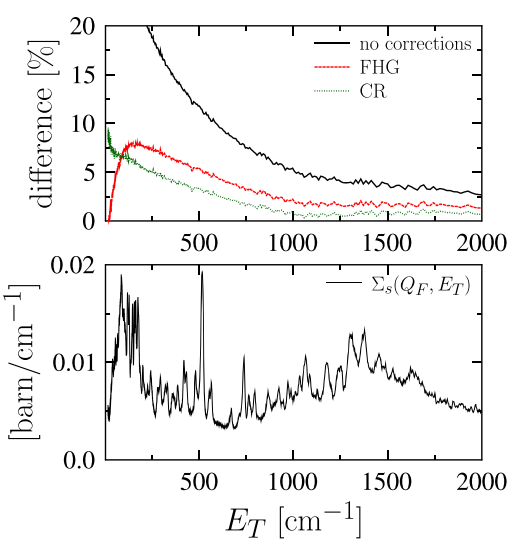

Figure 8. Forward-scattering INS spectrum of L-lysine (bottom panel), corrected using the experimental transmission signal and normalized to the absolute scale of barn $/ \mathrm{cm}^{-1}$ using the INSCorNorm algorithm. The relative differences between this spectrum and the one normalized without corrections (black line) or corrected using the nominal value of $n_{\mathrm{H}}$ and the FHG (red line) or the $\mathrm{CR}$ model (green line) are reported in the top panel.

512 forward-scattering signal and was obtained using the 513 experimental neutron transmission data collected on VESU514 VIO. For the normalization, we used the experimental value of $515\left\langle E_{\mathrm{k}}\right\rangle=149.2 \pm 0.5 \mathrm{meV}$, obtained by DINS measurements of 516 the same sample on VESUVIO. In the top panel of Figure 8, 517 we report the relative difference between the abovementioned 518 spectrum and the one normalized either without performing 519 self-attenuation corrections (black line) or using the nominal 520 density and the FHG (red dashed line) or CR model (green 521 dotted line). In this figure, a positive difference corresponds to 522 an underestimate of the vibrational intensity with respect to the correction procedure based on the experimental trans- 523 mission spectra. One can notice how, for $E_{\mathrm{T}} \simeq 500 \mathrm{~cm}^{-1}$, the 524 intensity of the vibrational modes is underestimated by about 525 $10 \%$ and increases more as one approaches the elastic line, in 526 the region where hydrogen-bonding fingerprints are expected. 527 When the data are corrected, either with the FHG or with the 528 CR models, the relative difference with the properly reduced 529 data is lower. In most of the energy range reported in the 530 figure, the CR model provides better results than the FHG 531 model, as one should expect from the organic nature of the 532 sample. In this case, the difference can be mainly related to the 533 lower value of the nominal hydrogen areal density with respect 534 to the effective one directly probed in the experimental 535 transmission data. It is interesting to notice in Figure 8 the 536 crossing point of the CR and FHG models at around 100537 $\mathrm{cm}^{-1}$. The existence of this crossing point, due to the different 538 dependence of $\sigma(E)$ in the two models upon the incident 539 neutron energy, had been anticipated in Figure 6.

540

It is important to note that atom-projected vibrational 541 densities of states are routinely used to evaluate anisotropic 542 displacement parameters (ADPs), for example, in molecular 543 crystals. ${ }^{61,62}$ Owing to the $1 / E_{\mathrm{T}}$ dependence of the ADPs, the 544 underestimate of the intensities of the modes in the lowest 545 energy range of the INS spectra is tantamount to a substantial 546 underestimate of the ADPs. Therefore, the INS spectra 547 corrected using the INSCorNorm algorithm provide robust 548 support to benchmarking phonon-based calculations of ADPs 549 and the related analysis of X-ray and neutron diffraction data. 550

\section{CONCLUSIONS}

In this work, the algorithm INSCorNorm has been described 551 and applied. It is a python-based procedure for the correction 552 and physical normalization of the INS spectra collected on the 553 TOSCA spectrometer. The algorithm can be used self- 554 consistently with the experimental transmission spectra, for 555 the corrections, and values of the proton mean kinetic energy, 556 for the normalization, measured on the VESUVIO spectrom- 557 eter. Alternatively, one can make use of two model functions 558 for the sample cross section of which one is suitable for organic 559 samples. A practical example for $\mathrm{ZrH}_{2}$ at $300 \mathrm{~K}$ has been 560 discussed. Moreover, we have applied the algorithm to INS 561 data from L-lysine, an organic system of broad interest in 562 medicine and biology, and we have discussed how properly 563 corrected spectra can provide a useful benchmark for 564 theoretical investigations probing intermolecular interactions 565 and predicting anisotropic displacement parameters. 566

While the results have been presented in the case of the 567 TOSCA spectrometer, the algorithm can be applied to results 568 from any inverted-geometry instrument. ${ }^{10}$ Importantly, next- 569 generation INS spectrometers ${ }^{63}$ would greatly benefit from 570 concurrent energy-selective neutron transmission measure- 571 ments over the same energy range probed in the vibrational 572 spectra. Such capability would allow a self-consistent 573 correction of the data using the experimental attenuation of 574 the sample, thus taking into account subtle dependencies upon 575 temperature and other sample-environment stimuli.

576

AUTHOR INFORMATION

Corresponding Author

Giovanni Romanelli - ISIS Facility, Rutherford Appleton Laboratory, Didcot, Oxfordshire OX11 OQX, U.K.;

- orcid.org/0000-0001-5963-4647;

Email: giovanni.romanelli@stfc.ac.uk
577 


\section{Authors}

584 Claudia Scatigno - Dipartimento di Scienze Fisiche e

585 Tecnologie della Materia, CNR, 00185 Roma, Italy; Museo

586 Storico della Fisica e Centro Studi e Ricerche Enrico Fermi,

$587 \quad 00184$ Rome, Italy

588 Enrico Preziosi - Dipartimento di Fisica e NAST Centre,

589 Università degli Studi di Roma "Tor Vergata", 00133 Rome,

$590 \quad$ Italy

591 Matteo Zanetti - Dipartimento di Scienze Fisiche e Tecnologie

592 della Materia, CNR, 00185 Roma, Italy; Advanced Nuclear

593 Systems, SCK-CEN, $2400 \mathrm{Mol}$, Belgium

594 Stewart F. Parker - ISIS Facility, Rutherford Appleton

595 Laboratory, Didcot, Oxfordshire OX11 0QX, U.K.;

596 orcid.org/0000-0002-3228-2570

597 Svemir Rudić - ISIS Facility, Rutherford Appleton

598 Laboratory, Didcot, Oxfordshire OX11 0QX, U.K.

599 Carla Andreani - Dipartimento di Fisica e NAST Centre,

600 Università degli Studi di Roma "Tor Vergata”, 00133 Rome,

$601 \quad$ Italy

602 Roberto Senesi - Dipartimento di Fisica e NAST Centre,

603 Università degli Studi di Roma "Tor Vergata", 00133 Rome,

604 Italy; CNR-IPCF, 98158 Messina, Italy

605 Complete contact information is available at:

606 https://pubs.acs.org/10.1021/acs.jctc.0c00790

607 Notes

608 The authors declare no competing financial interest.

\section{ACKNOWLEDGMENTS}

610 The authors gratefully acknowledge the financial support of 611 Regione Lazio (IR approved by Giunta Regionale n. G10795, 7 612 August 2019 published by BURL n. 6927 August 2019), 613 ISIS@MACH (I), and ISIS Neutron and Muon Source (UK) 614 of Science and Technology Facilities Council (STFC); the 615 financial support of Consiglio Nazionale delle Ricerche within 616 CNR-STFC Agreement 2014-2020 (N 3420), concerning 617 collaboration in scientific research at the ISIS Neutron and 618 Muon Source (UK) of Science and Technology Facilities 619 Council (STFC), and the ESS-ERIC for Instrument VESPA 620 Phase 1 are gratefully acknowledged.

\section{REFERENCES}

622 (1) Mitchell, P. C. H.; Parker, S. F.; Ramirez-Cuesta, A. J.; 623 Tomkinson, J. Vibrational spectroscopy with neutrons: with applications 624 in chemistry, biology, materials science and catalysis; World Scientific: 625 Singapore, 2005, DOI: 10.1142/5628

626 (2) Parker, S. F.; Carlile, C. J.; Pike, T.; Tomkinson, J.; Newport, R. 627 J.; Andreani, C.; Ricci, F. P.; Sacchetti, F.; Zoppi, M. TOSCA: a world 628 class inelastic neutron spectrometer. Phys. B 1997, 241-243, 154-156. 629 (3) ISIS Neutron and Muon Source, Science and Technology Facilities 630 Council, ISIS INS Database. 2020; https://www.isis.stfc.ac.uk/Pages/ 631 INS-database.aspx, Last access on July 2020

632 (4) Rudic, S.; Ramirez-Cuesta, A. J.; Parker, S. F.; Fernandez-Alonso, 633 F.; Pinna, R. S.; Gorini, G.; Salzmann, C. G.; McLain, S. E.; Skipper, 634 N. T. TOSCA international beamline review. RAL Technical Reports 635 RAL-TR-2013-015; STFC: 2013

636 (5) Pinna, R. S.; Rudić, S.; Parker, S. F.; Armstrong, J.; Zanetti, M.; 637 Skoro, G.; Waller, S. P.; Zacek, D.; Smith, C. A.; Capstick, M. J.; 638 McPhail, D. J.; Pooley, D. E.; Howels, G. D.; Gorini, G.; Fernandez639 Alonso, F. The neutron guide upgrade of the TOSCA spectrometer. 640 Nucl. Instrum. Methods Phys. Res., Sect. A 2018, 896, 68-74.

641 (6) Pinna, R. S.; Rudić, S.; Parker, S. F.; Gorini, G.; Fernandez642 Alonso, F. Monte carlo simulations of the TOSCA spectrometer:
Assessment of current performance and future upgrades. EPJ Web 643 Conf. 2015, 83, No. 03013.

(7) Parker, S. F.; Fernandez-Alonso, F.; Ramirez-Cuesta, A. J.; 645 Tomkinson, J.; Rudic, S.; Pinna, R. S.; Gorini, G.; Castañon, J. F. 646 Recent and future developments on TOSCA at ISIS. J. Phys.: Conf. 647 Ser. 2014, 554, No. 012003.

(8) Tomkinson, J.; Penfold, J.; Robertson, S. T. The optic modes in 649 $\mathrm{ZrH}_{2}$. RAL Technical Report RAL-89-074: 1989

650

(9) Couch, J. G.; Harling, O. K.; Clune, L. C. Structure in the 651 neutron scattering spectra of zirconium hydride. Phys. Rev. B 1971, 4, 652 2675.

(10) Colognesi, D. The high energy-transfer region in neutron 654 scattering vibrational spectra: What does it mean and what could it be 655 useful for? J. Neutron Res. 2017, 19, 147-167.

656

(11) West, G. B. Electron scattering from atoms, nuclei and 657 nucleons. Phys. Rep. 1975, 18, 263-323.

(12) Sears, V. F. Scaling and final-state interactions in deep-inelastic 659 neutron scattering. Phys. Rev. B 1984, 30, 44.

(13) Gunn, J. M. F.; Andreani, C.; Mayers, J. A new approach to 66 impulsive neutron scattering. J. Phys. C: Solid State Phys. 1986, 19, 662 L835.

(14) Andreani, C.; Colognesi, D.; Mayers, J.; Reiter, G. F.; Senesi, R. 664 Measurement of momentum distribution of light atoms and molecules 665 in condensed matter systems using inelastic neutron scattering. Adv. 666 Phys. 2005, 54, 377-469.

667

(15) Andreani, C.; Krzystyniak, M.; Romanelli, G.; Senesi, R.; 668 Fernandez-Alonso, F. Electron-volt neutron spectroscopy: beyond 669 fundamental systems. Adv. Phys. 2017, 66, 1-73.

(16) Andreani, C.; Senesi, R.; Krzystyniak, M.; Romanelli, G.; 671 Fernandez-Alonso, F. Atomic quantum dynamics in materials 672 research. Exp. Methods Phys. Sci. 2017, 49, 403-457. 673

(17) Senesi, R.; Andreani, C.; Bowden, Z.; Colognesi, D.; Degiorgi, 674 E.; Fielding, A. L.; Mayers, J.; Nardone, M.; Norris, J.; Praitano, M.; 675 Rhodes, N. J.; Stirling, W. G.; Tomkinson, J.; Uden, C. VESUVIO: a 676 novel instrument for performing spectroscopic studies in condensed 677 matter with eV neutrons at the ISIS facility. Phys. B 2000, 276-278, 678 200-201.

(18) Andreani, C.; Pietropaolo, A.; Senesi, R.; Gorini, G.; Tardocchi, 680 M.; Bracco, A.; Rhodes, N.; Schooneveld, E. Electron-volt spectros- 681 copy at a pulsed neutron source using a resonance detector technique. 682 Nucl. Instrum. Methods Phys. Res., Sect. A 2002, 481, 509-520. 683

(19) Gorini, G.; Perelli-Cippo, E.; Tardocchi, M.; Andreani, C.; 684 D’Angelo, A.; Pietropaolo, A.; Senesi, R.; Imberti, S.; Bracco, A.; 685 Previtali, E.; Pessina, G.; Rhodes, N. J.; Schooneveld, E. M. The 686 resonant detector and its application to epithermal neutron 687 spectroscopy. Nucl. Instrum. Methods Phys. Res., Sect. A 2004, 529, 688 293-300.

689

(20) Mayers, J.; Tomkinson, J.; Abdul-Redah, T.; Stirling, W. G.; 690 Andreani, C.; Senesi, R.; Nardone, M.; Colognesi, D.; Degiorgi, E. 691 VESUVIO-the double difference inverse geometry spectrometer at 692 ISIS. Phys. B 2004, 350, E659-E662.

(21) Pietropaolo, A.; Andreani, C.; Filabozzi, A.; Senesi, R.; Gorini, 694 G.; Perelli-Cippo, E.; Tardocchi, M.; Rhodes, N. J.; Schooneveld, E. 695 M. DINS measurements on VESUVIO in the Resonance Detector 696 configuration: proton mean kinetic energy in water. J. Instrum. 2006, 697 1, P04001.

(22) Mayers, J.; Reiter, G. The VESUVIO electron volt neutron 699 spectrometer. Meas. Sci. Technol. 2012, 23, No. 045902.

(23) Rodríguez Palomino, L. A.; Dawidowski, J.; Márquez Damián, 701 J. I.; Cuello, G. J.; Romanelli, G.; Krzystyniak, M. Neutron total cross- 702 section of hydrogenous and deuterated 1-and 2-propanol and n- 703 butanol measured using the VESUVIO spectrometer. Nucl. Instrum. 704 Methods Phys. Res., Sect. A 2017, 870, 84-89.

(24) Romanelli, G.; Rudić, S.; Zanetti, M.; Andreani, C.; Fernandez- 706 Alonso, F.; Gorini, G.; Krzystyniak, M.; Skoro, G. Measurement of the 707 para-hydrogen concentration in the ISIS moderators using neutron 708 transmission and thermal conductivity. Nucl. Instrum. Methods Phys. 709 Res., Sect. A 2018, 888, 88-95. 
711 (25) Capelli, S. C.; Romanelli, G. An effective hydrogen scattering 712 cross section for time-of-flight neutron experiments with simple 713 organic molecules. J. Appl. Crystallogr. 2019, 52, 1233-1237.

714 (26) Rodríguez Palomino, L. A.; Dawidowski, J.; Helman, C.; 715 Márquez Damián, J. I.; Romanelli, G.; Krzystyniak, M.; Rudić, S.; 716 Cuello, G. J. Determination of the scattering cross section of calcium 717 using the VESUVIO spectrometer. Nucl. Instrum. Methods Phys. Res., 718 Sect. A 2019, 927, 443-450.

719 (27) Romanelli, G.; Krzystyniak, M.; Senesi, R.; Raspino, D.; Boxall, 720 J.; Pooley, D.; Moorby, S.; Schooneveld, E.; Rhodes, N. J.; Andreani, 721 C.; Fernandez-Alonso, F. Characterisation of the incident beam and 722 current diffraction capabilities on the VESUVIO spectrometer. Meas. 723 Sci. Technol. 2017, 28, No. 095501.

724 (28) Robledo, J. I.; Dawidowski, J.; Márquez Damián, J. I.; Škoro, 725 G.; Bovo, C.; Romanelli, G. Measurement of neutron total cross 726 sections at the VESUVIO spectrometer. Nucl. Instrum. Methods Phys. 727 Res., Sect. A 2020, 164096.

728 (29) Krzystyniak, M.; Romanelli, G.; Fabian, M.; Gutmann, M.; 729 Festa, G.; Arcidiacono, L.; Gigg, M.; Drubicki, K.; Andreani, C.; 730 Senesi, R.; Fernandez-Alonso, F. VESUVIO+: The Current Testbed 731 for a Next-generation Epithermal Neutron Spectrometer. J. Phys.: 732 Conf. Ser. 2018, 1021, No. 012026.

733 (30) Evans, A. C.; Timms, D. N.; Mayers, J.; Bennington, S. M. 734 Neutron-scattering study of the impulse approximation in $\mathrm{ZrH}_{2}$. Phys. 735 Rev. B 1996, 53, 3023.

736 (31) Scatigno, C.; Zanetti, M.; Rudic, S.; Gorini, G.; Fernandez737 Alonso, F.; Andreani, C.; Senesi, R. Quantitative Benchmarks of state738 of-art Neutron Chemical Spectrometers - Part 1: TOSCA. ISIS 739 Neutron and Muon Source Data Journal; STFC ISIS Neutron and 740 Muon Source: 2018.

741 (32) Nelson, D.; Cox, M. Lehninger Principles of Biochemistry; (4th 742 Ed.); W.H. Freeman and Company: New York, 2005

743 (33) Wang, Z. A.; Cole, P. A. The Chemical Biology of Reversible 744 Lysine Post-translational Modifications. Cell Chem. Biol. 2020, 953.

745 (34) Feuz, L.; Strunz, P.; Geue, T.; Textor, M.; Borisov, O. 746 Conformation of poly (L-lysine)-graft-poly (ethylene glycol) molec747 ular brushes in aqueous solution studied by small-angle neutron 748 scattering. Eur. Phys. J. E 2007, 23, 237-245.

749 (35) Zanetti, M.; Gorini, G.; Romanelli, G.; Scatigno, C.; Andreani, 750 C.; Senesi, R.; Fernandez-Alonso, F. Quantitative Benchmarks of 751 state-of-art Neutron Chemical Spectrometers - Part 2: VESUVIO. 752 ISIS Neutron and Muon Source Data Journal; STFC ISIS Neutron and 753 Muon Source: 2018.

754 (36) Pinna, R. S.; Rudić, S.; Capstick, M. J.; McPhail, D. J.; Pooley, 755 D. E.; Howells, G. D.; Gorini, G.; Fernandez-Alonso, F. Detailed 756 characterisation of the incident neutron beam on the TOSCA 757 spectrometer. Nucl. Instrum. Methods Phys. Res., Sect. A 2017, 870, 758 79-83.

759 (37) Pietropaolo, A.; Andreani, C.; Rebai, M.; Giacomelli, L.; Gorini, 760 G.; Perelli Cippo, E.; Tardocchi, M.; Fazzi, A.; Verona Rinati, G.; 761 Verona, C.; Marinelli, M.; Milani, E.; Frost, C. D.; Schooneveld, E. M. 762 Fission diamond detectors for fast-neutron ToF spectroscopy. 763 Europhys. Lett. 2011, 94, 62001.

764 (38) Tardocchi, M.; Pietropaolo, A.; Andreani, C.; Bracco, A.; 765 D’Angelo, A.; Gorini, G.; Imberti, S.; Senesi, R.; Rhodes, N. J.; 766 Schooneveld, E. M. Cadmium-Zinc-Telluride photon detector for 767 epithermal neutron spectroscopy-pulse height response character768 isation. Nucl. Instrum. Methods Phys. Res., Sect. A 2004, 526, 477-492. 769 (39) Andreani, C.; Pietropaolo, A.; Senesi, R.; Gorini, G.; Perelli770 Cippo, E.; Tardocchi, M.; Rhodes, N.; Schooneveld, E. M. A resonant 771 detector for high-energy inelastic neutron scattering experiments. 772 Appl. Phys. Lett. 2004, 85, 5454.

773 (40) Andreani, C.; Romanelli, G.; Senesi, R. Direct Measurements of 774 Quantum Kinetic Energy Tensor in Stable and Metastable Water near 775 the Triple Point: An Experimental Benchmark. J. Phys. Chem. Lett. 776 2016, 7, 2216-2220.

777 (41) Andreani, C.; Senesi, R.; Krzystyniak, M.; Romanelli, G.; 778 Fernandez-Alonso, F. Experimental studies of nuclear quantum effects in condensed matter: The case of water. Riv. Nuovo Cimento 2018, 41, 779 $291-340$

(42) Romanelli, G.; Senesi, R.; Zhang, X.; Loh, K. P.; Andreani, C. 781 Probing the effects of $2 \mathrm{D}$ confinement on hydrogen dynamics in 782 water and ice adsorbed in graphene oxide sponges. Phys. Chem. Chem. 783 Phys. 2015, 17, 31680-31684.

(43) Romanelli, G.; Liscio, A.; Senesi, R.; Zamboni, R.; Treossi, E.; 785 Liscio, F.; Giambastiani, G.; Palermo, V.; Fernandez-Alonso, F.; 786 Andreani, C. Soft confinement of water in graphene-oxide 787 membranes. Carbon 2016, 108, 199-203.

788

(44) De Michele, V.; Romanelli, G.; Cupane, A. Dynamics of 789 supercooled confined water measured by deep inelastic neutron 790 scattering. Front. Phys. 2018, 13, 138205.

(45) Agrawal, A. K. Multiple scattering of neutrons in gaseous and 792 liquid methane. Phys. Rev. A 1971, 4, 1560.

(46) Fernandez-Alonso, F.; Price, D. L. Neutron Scattering; Academic 794 Press: 2013

(47) Watson, G. I. Neutron compton scattering. J. Phys.: Condens. 796 Matter 1996, 8, 5955.

(48) Pace, E.; Salmè, G.; West, G. Final state interaction in quasi- 798 elastic electron scattering by nuclei and y scaling. Phys. Lett. B 1991, 799 273, 205-210.

(49) Arnold, O.; Bilheux, J. C.; Borreguero, J. M.; Buts, A.; 801 Campbell, S. I.; Chapon, L.; Doucet, M.; Draper, N.; Ferraz Leal, R.; 802 Gigg, M. A.; Lynchc, V. E.; Markvardsena, A.; Mikkelsonec, D. J.; 803 Mikkelsonec, R. L.; Millerf, R.; Palmena, K.; Parkera, P.; Passosa, G.; 804 Zikovskyc, J. J. Mantid-Data analysis and visualization package for 805 neutron scattering and $\mu$ SR experiments. Nucl. Instrum. Methods Phys. 806 Res., Sect. A 2014, 764, 156-166.

(50) Romanelli, G.; Hewer, B.; Krzystyniak, M.; Gigg, M.; 808 Tolchenov, R.; Mukhopadhyay, S.; Fernandez-Alonso, F. Data 809 analysis of neutron Compton scattering experiments using MANTID. 810 J. Phys.: Conf. Ser. 2018, 1055, No. 012016.

(51) Pietropaolo, A.; Andreani, C.; D’Angelo, A.; Senesi, R.; Gorini, 812 G.; Imberti, S.; Tardocchi, M.; Rhodes, N.; Schooneveld, E. S. $\gamma 813$ detectors for Deep Inelastic Neutron Scattering in the 1-100 eV 814 energy region. Appl. Phys. A 2002, 74, s189-s190.

(52) Pietropaolo, A.; Perelli Cippo, E.; Gorini, G.; Tardocchi, M.; 816 Schooneveld, E. M.; Andreani, C.; Senesi, R. $\gamma$-Ray background 817 sources in the VESUVIO spectrometer at ISIS spallation neutron 818 source. Nucl. Instrum. Methods Phys. Res., Sect. A 2009, 608, 121-124. 819 (53) Schooneveld, E. M.; Mayers, J.; Rhodes, N. J.; Pietropaolo, A.; 820 Andreani, C.; Senesi, R.; Gorini, G.; Perelli-Cippo, E.; Tardocchi, M. 821 Foil cycling technique for the VESUVIO spectrometer operating in 822 the resonance detector configuration. Rev. Sci. Instrum. 2006, 77, 823 No. 095103.

(54) Mayers, J.; Fielding, A. L.; Senesi, R. Nucl. Instrum. Methods 825 Phys. Res., Sect. A 2002, 481, 454. 826

(55) Andreani, C.; Baciocco, G.; Holt, R. S.; Mayers, J. Resolution in 827 deep inelastic neutron scattering using pulsed neutron sources. Nucl. 828 Instrum. Methods Phys. Res., Sect. A 1989, 276, 297-305. 829

(56) Imberti, S.; Andreani, C.; Garbuio, V.; Gorini, G.; Pietropaolo, 830 A.; Senesi, R.; Tardocchi, M. Resolution of the VESUVIO 831 spectrometer for High-energy Inelastic Neutron Scattering experi- 832 ments. Nucl. Instrum. Methods Phys. Res., Sect. A 2005, 552, 463.

(57) Pietropaolo, A.; Andreani, C.; Filabozzi, A.; Pace, E.; Senesi, R. 834 Resolution function in deep inelastic neutron scattering using the Foil 835 Cycling Technique. Nucl. Instrum. Methods Phys. Res., Sect. A 2007, 836 570, 498-510.

837

(58) Prisk, T. R.; Kolesnikov, A. I.; Granroth, G. E.; Lin, J.-L.; 838 Heuser, B. J. Vibrational modes and quantum zero-point energy of 839 hydrogen in $\mathrm{ZrH} 0.0155$ and $\mathrm{ZrH} 2$. J. Alloys Compd. 2020, 818, 840 152832 .

(59) Boeckx, B.; Maes, G. Experimental and Theoretical 842 Observation of Different Intramolecular H-bonds in Lysine 843 Conformations. J. Phys. Chem. B 2012, 116, 12441-12449. 844

(60) Stearns, J. A.; Seaiby, C.; Boyarkin, O. V.; Rizzo, T. R. 845 Spectroscopy and conformational preferences of gas-phase helices. 846 Phys. Chem. Chem. Phys. 2009, 11, 125-132. 
848 (61) Madsen, A. Ø.; Civalleri, B.; Ferrabone, M.; Pascale, F.; Erba, 849 A. Anisotropic displacement parameters for molecular crystals from 850 periodic Hartree-Fock and density functional theory calculations. 851 Acta Crystallogr., Sect. A: Found. Crystallogr. 2013, 69, 309-321.

852 (62) George, J.; Wang, A.; Deringer, V. L.; Wang, R.; Dronskowski, 853 R.; Englert, U. Anisotropic displacement parameters from dispersion854 corrected DFT methods and their experimental validation by 855 temperature-dependent X-ray diffraction. CrystEngComm 2015, 17, $8567414-7422$.

857 (63) Zanetti, M.; Bellissima, S.; del Rosso, L.; Masi, F.; Chowdhury, 858 M.; De Bonis, A.; Di Fresco, L.; Scatigno, C.; Armstrong, J.; Rudić, S.; 859 Parker, S. F.; Hartl, M.; Colognesi, D.; Senesi, R.; Andreani, C.; 860 Gorini, G.; Fernandez-Alonso, F. Neutronic developments on 861 TOSCA and VESPA: Progress to date. Phys. B 2019, 562, 107-111. 